\title{
X. Baur
}

\section{Auswirkungen der Belastungen unter Tage im Steinkohlenbergbau auf die Lunge}

\author{
Effects on the Lung Due to Underground Coal Mining Work
}

\section{Zusammenfassung}

Die langjährige inhalative Exposition gegenüber Stäuben im Steinkohlenbergbau kann zu verschiedenen pathologischen Lungenveränderungen führen, insbesondere zu einer chronischen Bronchitis ohne und mit obstruktiver Ventilationsstörung, einem Lungenemphysem, einer Pneumokoniose (KohlenbergarbeiterPneumokoniose, in Deutschland unter Silikose subsumiert). Die vorgenannten Gesundheitsstörungen stehen z.T. in einem engen pathogenetischen und pathophysiologischen Zusammenhang und sind nicht als verschiedene Entitäten aufzufassen. In der Regel zeigen langjährige Exponierte mehr oder weniger alle diese angeführten Pathologica. Eine Sonderform stellt die Silicotuberkulose dar. Aufgrund individueller (genetischer?) Dispositionsfaktoren ist das Ausmaß der Lungenveränderungen sehr unterschiedlich. Neben weit gehend resistenten Personen trifft man Patienten mit ausgeprägten Krankheitsbildern wie einer schwieliger Silikose, einem massiven Emphysem (bis hin zur sog. „schwarzen Löcherlunge“), oder dem Caplan-Syndrom an. In mehreren Studien konnte gezeigt werden, dass der pathologisch verifizierte Fibrosegrad mit dem in der Lunge abgelagerten kristallinen $\mathrm{SiO}_{2}$-Gehalt assoziiert ist, während das Emphysem mit der inkorporierten Kohlenstaubmenge korreliert. Für die Diagnostik und Begutachtung sind von Bedeutung, dass die konventionelle Radiologie eine geringe Sensitivität für den Nachweis von Silikosegranulomen aufweist und dass versicherungsrechtlich relevante Gesundheitsstörungen mit MdE des langjährig unter Tage tätigen Bergmannes ohne (BK 4111 falls nach dem 31.12.1992 aufgetreten) und mit Silikose (auch bei Kategorien $<2 / 3$ ) nicht selten bestehen.

\section{Abstract}

The long-term exposure to dust in the hard coal mining industry can lead to various pathological lung changes, especially to chronic bronchitis without and with obstructive ventilation disorder, lung emphysema, pneumoconiosis (coal miner's pneumoconiosis, in Germany categorized as silicosis) and silicotuberculosis. These health disorders show a close pathogenetic and pathophysiological association and should not necessarily be regarded as individual entities. Most exposed subjects demonstrate more or less all of these pathological disorders. On account of individual (genetic?) susceptibility, their degree differs greatly. Some individuals are largely resistent, other subjects show severe effects like emphysema, progressive massive pneumoconiosis, or the Caplan syndrome. Several studies showed that the pathologically verified degree of lung fibrosis is associated with lung crystalline $\mathrm{SiO}_{2}$ content whereas the emphysema score is inversely correlated with the coal content. With regard to diagnostics and medical expert opinion, it is important that conventional radiology has a low sensitivity. Further, health impairments of miners engaged for longtime which are insurance relevant (MdE) exist in cases without (BK 4111 if beginning after 12/31/1992) or with coalworkers' pneumoconiosis even for categories $<2 / 3$. 


\section{Prävalenz der chronischen Bronchitis unter britischen Steinkohlenbergleuten (\%)}

Eingeatmeter und in den Alveolen und Atemwegen abgelagerter schwer löslicher Staub mit kristallinem $\mathrm{SiO}_{2}$, wie er im Steinkohlenbergbau auftritt, akkumuliert in der Lunge, wenn die Clearancerate des Atemwegsepithels überschritten wird [47] bei einer Konzentration des alveolengängigen Staubs von etwa $1 \mathrm{mg} / \mathrm{m}^{3}$. Die mittlere Halbwertzeit dieses deponierten Staubs beträgt in tierexperimentellen Befunden 6-12 Monate. Die Staubpartikel werden von Makrophagen phagozytiert und in das Lungeninterstitium transportiert. Dabei kommt es zur Makrophagen-Aktivierung und -Proliferation mit Bildung von inflammatorischen und zytotoxischen Zytokinen, Zellwachstumsfaktoren sowie reaktiven Sauerstoffspezies. Die phagozytierenden Zellen gehen schließlich an den nicht abbaubaren Staubpartikeln unter Freisetzung auch lytischer Enzyme zugrunde. Immer neue Makrophagen nehmen die Partikel auf und setzen den Kreislauf fort. Die Folgen sind eine Chronifizierung des Entzündungsprozesses mit Involvierung von Neutrophilen, Epithelzellen und Fibroblasten, die aktiviert werden. Dabei findet eine gesteigerte Kollagenfaser-Synthese statt. In-vivo- und In-vitro-Untersuchungen zeigen, dass Kohlengrubenstaub die Expression von LeukozytenAktivierungsfaktoren, monozyten-chemotaktisches Protein, TNF $\alpha$ sowie Neutrophilen-Adhäsionsfaktoren (ICAM) aufreguliert. Die genannten pathophysiologischen Vorgänge führen schließlich zu einer Schädigung der Lungen-Zielzellen und wichtiger Makromoleküle wie $\alpha_{1}$-Antitrypsin und der DNS [60]. Im interstitiellen Bindegewebe können sich silikotische Herde bilden, die einen typischen Aufbau mit zentraler Hyalinisierung und konzentrisch-lamellenartiger Schichtung aufweisen. Als Besonderheit der Pneumokoniose des Steinkohlenbergmanns, der kohlenstoffreichem Staub ausgesetzt ist, findet sich ein breiter Saum von staubenthaltenden Makrophagen um die silikotischen Knötchen. Gelegentlich zu beobachtende Autoantikörper stellen in der Regel Epiphänomene dar. In sehr seltenen Fällen ist eine besondere großknotige Silikoseform mit einer rheumatoiden $\mathrm{Ar}$ thritis assoziiert (Caplan-Syndrom). Die morphologischen und funktionellen Veränderungen im Sinne einer Restriktion, Atemwegsobstruktion und eines Lungenemphysems gehen mit Krankheitssymptomen, röntgenologisch fassbaren Pathologica (vorwiegend rundlichen Lungenschatten, Schwielen und/oder Emphysem), erhöhter Morbidität (v.a. chronische Bronchitis mit und ohne Lungenfunktionseinschränkung) und Mortalität einher, wodurch es häufig zu einer relevanten Minderung der Erwerbsfähigkeit (MdE) kommt. Im Folgenden soll eine Übersicht des gegenwärtigen Kenntnisstandes hierzu gegeben werden.

\section{Ergebnisse}

\section{Chronische Bronchitis}

Marine u. Mitarb. [41] fanden im „Pneumoconiosis Field Research" des National Coal Bords in Großbritannien, dem bisher umfangreichsten epidemiologischen Forschungsprogramm im Steinkohlenbergbau, eine signifikante positive Assoziation zwischen der kumulierten Feinstaubdosis und dem Auftreten einer chronischen Bronchitis im Sinne der WHO-Definition (Abb.1). Eine solche Beziehung existiert auch für die chronische obstruktive Bronchitis wie u.a. die vorgenannte Untersuchung sowie die in den 60er-Jahren durchgeführte DFG-Studie zeigen (Abb. 2a,

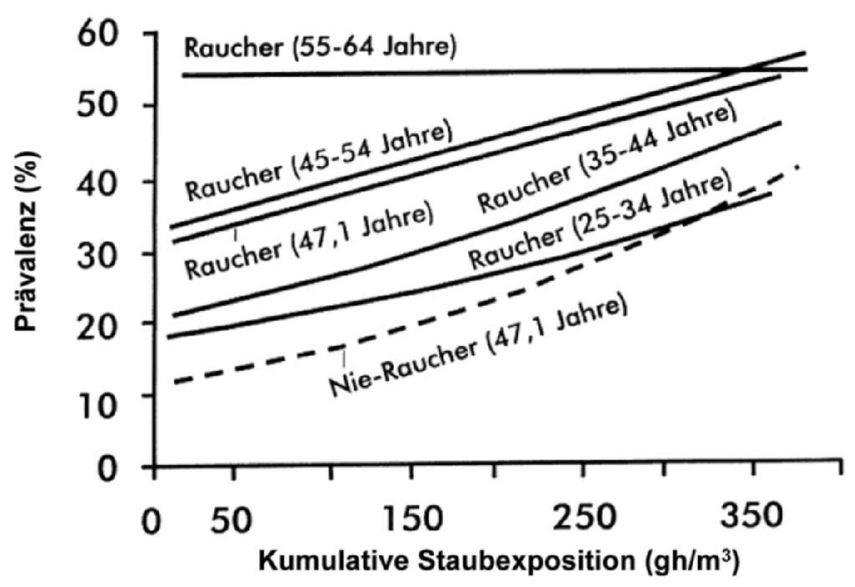

Quelle: Marine et al.; Am. Rev. Resp. Dis. 1988, 137, 106

Abb. 1 Prävalenz der chronischen Bronchitis unter britischen Steinkohlenbergleuten, abgeleitet von logistischen Regressionsgleichungen unter Berücksichtigung der kumulativen Staubexposition [41]. $R=$ Raucher; NR = Nichtraucher; J = Jahre.

2b). Bereits für durchschnittliche Konzentrationen alveolengängigen Staubs unter $1 \mathrm{mg} / \mathrm{m}^{3}$ waren nach langjähriger Exposition relevante Exzessrisiken objektivierbar [35]. Die DFG-Studie ergab für die komplexe anamnestisch-klinische und lungenfunktionsanalytische Zielgröße „chronische bronchiale Reaktion“ $\left(\mathrm{K}_{\mathrm{ov}}\right.$; Mindestpunktzahl =2) unabhängig vom Alter und vom Rauchverhalten eine signifikante Assoziation mit der durchschnittlichen Staubkonzentration im deutschen Steinkohlenbergbau $[18,20,36]$ Im US-amerikanischen Steinkohlenbergbau wurden gleichartige Zusammenhänge beschrieben [27]. Seixas u. Mitarb. (Tab. 5) schätzten mit einem logistischen Modell Risiken für bronchitische Symptome. Für die chronische obstruktive Bronchitis erhielten sie eine Verdopplung des Risikos bei einer alveolengängigen Staubdosis von $20 \mathrm{mgm}^{-3} \mathrm{a}$ (entspricht ca. $\left.32 \mathrm{ghm}^{-3}\right)\left(\mathrm{mgm}^{-3}\right.$ und $\mathrm{ghm}^{-3}$ sind Produkte aus mittlerer Konzentration in $\mathrm{mg}$ bzw. $\mathrm{g}$ pro $\mathrm{m}^{3}$ und Jahren bzw. Stunden).

\section{Radiologische Lungenbefunde}

Reisner [56], Reisner und Robock [54] und Reisner u. Mitarb. [55] haben bereits seit den 70er-Jahren Zusammenhänge zwischen der Staubdosis im Steinkohlenbergbau und der Entwicklung der radiologisch fassbaren Silikose festgestellt (Abb.3). Zu entsprechenden Resultaten kamen Piontek u. Mitarb. [51] und Morfeld [45], die außerdem einen stärkeren silikogenen Einfluss von stratigrafisch älteren Horizonten (höherer Inkohlungsgrad) beschrieben. Positive Dosis-Wirkungs-Beziehungen zwischen Staubdosis und Silikosehäufigkeit wurden auch von Hurley u. Mitarb. 1982, Hurley u. Mitar. [29] und Maclaren [28] und Collins u. Mitarb. [15] in ihren Auswertungen des Pneumoconiosis Field Research im britischen Steinkohlenbergbau beobachtet (Tab. 1).

Nach Collins u. Mitarb. [15] war das Auftreten von kleinen rundlichen bzw. unregelmäßigen Lungenschatten - in einem multivariaten logistischen Modell unter Berücksichtigung des Rauchverhaltens und des Alters - mit der kumulierten Belastung gegenüber alveolengängigem Kohlengrubenstaub assoziiert (Re- 

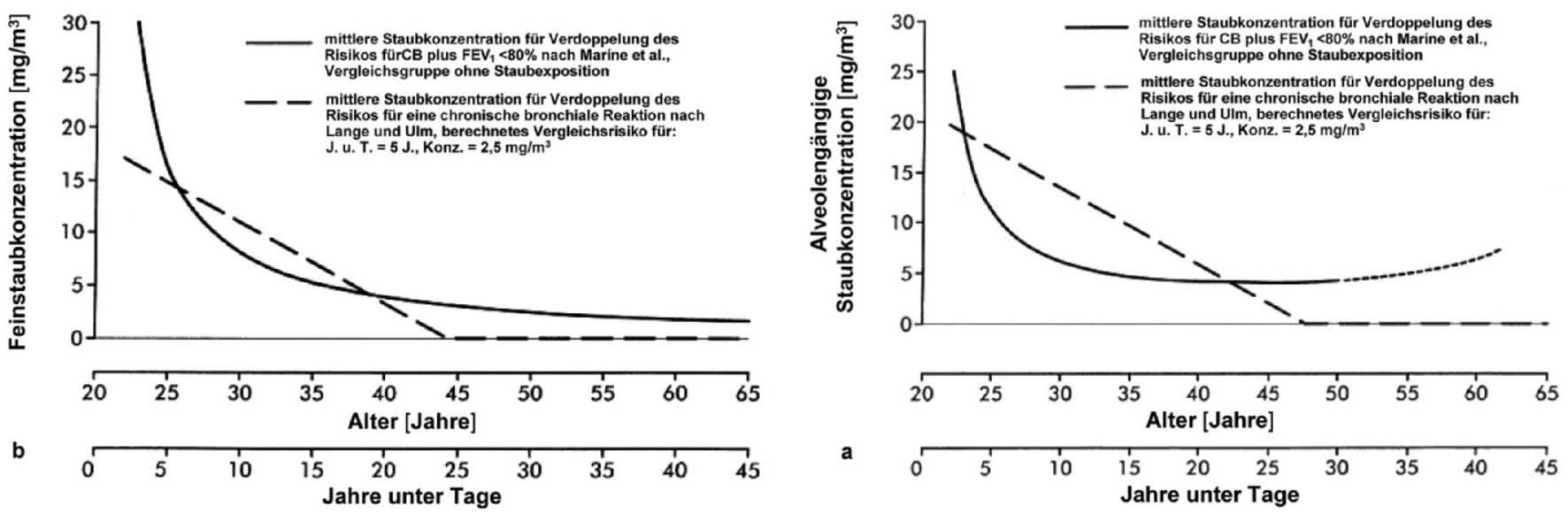

Abb. 2 Konzentration des alveolengängigen Kohlengrubenstaubs, die - in Abhängigkeit vom Alter - zu einer Häufigkeitsverdopplung einer chronischen obstruktiven Bronchitis führt [7,8]; a) Nieraucher, b) Raucher

gressionskoeffizient (Standardabweichung) pro $100 \mathrm{ghm}^{-3} 0,975$ [0,103] bzw. 0,5773 [0,133]). Gleiches gilt im Prinzip für Stäube mit hohem Quarzgehalt, wie sie in Erzbergwerken und Goldminen, im Tunnelbau, in Gießereien, Steinbrüchen, in steinverarbeitenden Betrieben, in der Glas- und Keramikindustrie, beim Sandstrahlen etc. vorkommen [12,23,25,42,48].

Unter den heute wesentlich verbesserten arbeitshygienischen Bedingungen im deutschen Steinkohlenbergbau treten höhergradige Silikosen nicht mehr auf, auch deshalb, weil die Arbeitseinsatzlenkung empfindliche Personen aus der hohen Belastung herausnimmt [17]. Seltenere radiologisch-klinische Diagnosen unter Steinkohlenbergleuten stellen das Caplan-Syndrom (großknotige Silikose mit rheumatoider Arthritis) und die Silikotuberkulose (BK 4102) dar. Das Risiko für letztere ist v.a. bei fortgeschrittener Silikose erhöht.

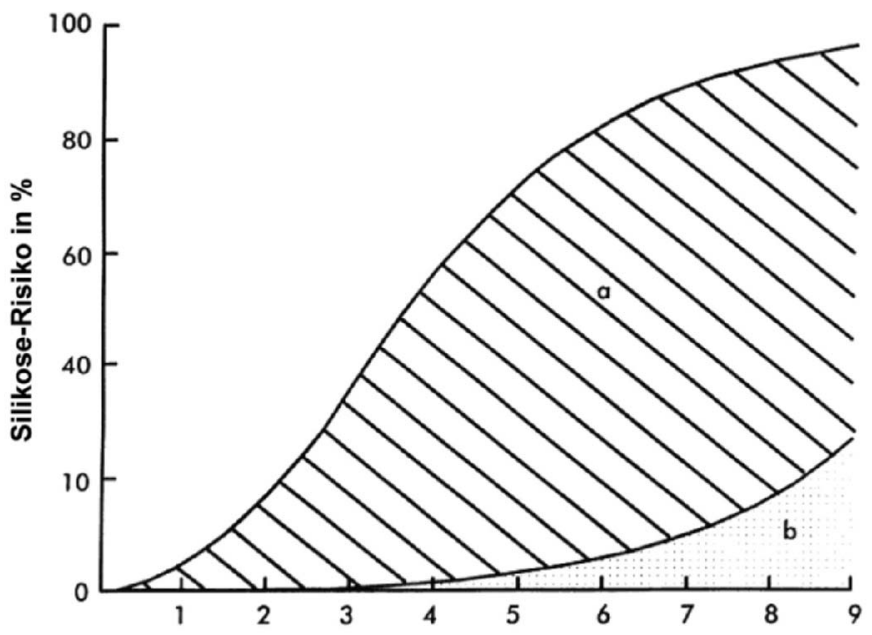

Mittlere Konzentration des alveolengängigen Staubs in $\mathrm{mg} / \mathrm{m}^{3}$

Abb. 3 Zusammenhang zwischen der mittleren Konzentration des alveolengängigen Staubs im deutschen Steinkohlenbergbau (35 Arbeitsjahre) und dem Risiko, eine Silikose der Kategorie $\leq 1$ (a) oder $>1$ bzw. Schwielenbildung (b) zu entwickeln [56].

\section{Lungenfunktion}

\section{Atemwegswiderstand $\boldsymbol{R}_{t}$}

In der Literatur finden sich etwas divergierende Ergebnisse in Abhängigkeit vom röntgenologischen Lungenbefund. Zum Teil wird eine im Mittel erhöhte Atemwegsresistance unter deutschen Steinkohlenbergleuten ohne Silikose beschrieben [68]. Andererseits teilen Ulmer u. Mitarb. [69] mit, dass erst höhergradige Silikosen (B- und C-Schwielen) mit einer eindeutigen Überhäufigkeit obstruktiver Atemwegserkrankungen einhergehen (Abb. 4). Letztere Darstellung war letztendlich die Basis der sog. „Moerser Konvention“, nach der bisher Silikosen in der Regel erst ab einer Kategorie von 3/2 bzw. 2/3 [32,33] als Berufskrankheit entschädigt werden; bei schwächerer Silikoseausprägung wurde nicht von einer Überhäufigkeit pathologischer Lungenfunktionsbefunde ausgegangen.

Tab. 1 Prävalenz verschiedener, radiologisch definierter SilikoseKategorien (Pneumokoniose der Steinkohlenbergleute) in Abhängigkeit von der durchschnittlichen Feinstaubkonzentration von $1-3 \mathrm{mg} / \mathrm{m}^{3}[21,28]$

\begin{tabular}{|c|c|c|c|c|}
\hline \multirow{2}{*}{$\begin{array}{l}\text { Feinstaub- } \\
\text { Konz. } \\
\left(\mathrm{mg} / \mathrm{m}^{3}\right)\end{array}$} & \multicolumn{3}{|c|}{$\begin{array}{l}\text { Röntgenklassifikation } \\
\text { nicht schwielige Silikose; } \\
\text { Kategorie: }\end{array}$} & \multirow{2}{*}{$\begin{array}{l}\text { schwielige } \\
\text { Silikose; } \\
\text { A, B, C } \\
\text { Prävalenz (\%) }\end{array}$} \\
\hline & \multicolumn{3}{|c|}{ Prävalenz (\%) } & \\
\hline 1 & 2,60 & 0,68 & 0,05 & 0,25 \\
\hline 2 & 5,94 & 1,63 & 0,15 & 0,64 \\
\hline 3 & 9,40 & 2,68 & 0,27 & 1,10 \\
\hline
\end{tabular}




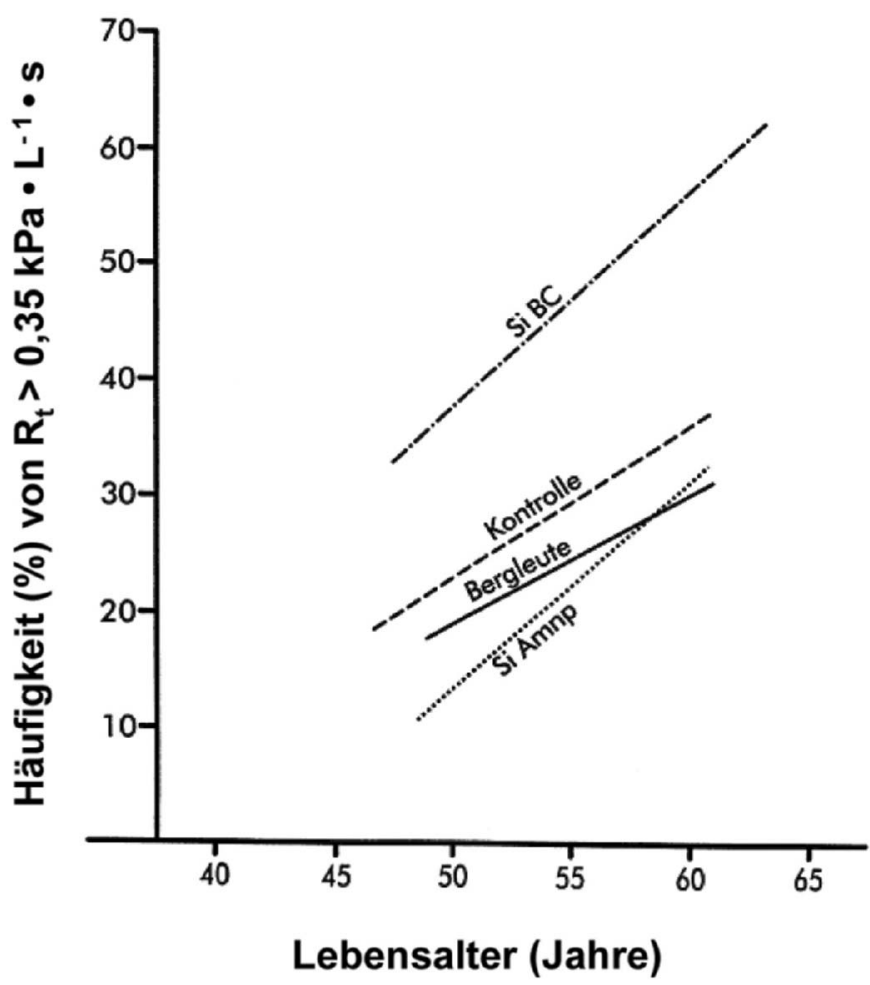

Abb. 4 Häufigkeit erhöhter Atemwegswiderstände (>0,35 $\mathrm{kPa} \times$ $\mathrm{L}^{-1} \times \mathrm{s}$ ) unter Steinkohlenbergleuten ohne und mit schwieliger Silikose [34] in Abhängigkeit vom Lebensalter [69].

\section{Spirometrische Lungenfunktionsparameter}

Im Vergleich zu Kontrollkollektiven haben im deutschen Steinkohlenbergbau unter Tage Beschäftigte signifikant niedrige Werte von VC, FEV , FRC und RV, und zwar auch dann, wenn noch keine radiologisch fassbare Silikose vorliegt. Mit zunehmender Silikosekategorie verstärkt sich die Funktionsstörung in der Regel. So liegen beispielsweise die Mittelwerte der VC von 50- bis 60-jährigen Bergleuten nach Smidt ([63]; Tab. 2a) um ca. $600 \mathrm{~mL}$ bzw. $800 \mathrm{~mL}$ (ohne Silikose und Silikose-Kategorie 1 bzw. Silikose-Kategorie 2 -3) unterhalb der nicht im Bergbau tätigen Kontrollgruppe (Tab. 2a). Für die Mittelwerte der $\mathrm{FEV}_{1}$ betragen die entsprechenden Unterschiede 370 - 640 mL bzw. 770 mL. Die Reduktion der Lungenfunktion ist damit bei Smidt [63] überschlägig knapp 30 - 45\% stärker als der Abfall vom Sollmittelwert zum Sollgrenzwert ( = 1,64 × RSD; [52]).

Zu ähnlichen Ergebnissen kommen Ulmer u. Mitarb. [68] (Tab. 2b) und Carstens u. Mitarb. [14] (Tab. 2c).

Carstens u. Mitarb. [14] weisen außerdem darauf hin, dass junge Bergleute bis zum Alter von 30 Jahren den Sollmittelwert der VC wesentlich übertreffen; hier macht sich ein Healthy-Worker-Effekt bemerkbar. Jenseits des 50. Lebensjahres beträgt der VC-Verlust der Durchschnittbevölkerung 12\%, der gesunden Bergleute aber 37\%, der Bergleute mit Emphysembronchitis 39\% und jener der an Silikose Erkrankten 42\%. Carstens u. Mitarb. beobachteten dabei vor allem eine Abnahme des inspiratorischen Anteils der Vitalkapazität („Reserveluft“), die von 35 auf $28 \%$ unter den über 50-Jährigen abnimmt.
Der Hauptverlust der Lungenfunktion entsteht nach allen diesen Studien aus dem deutschen Steinkohlenbergbau durch die kumulierte Belastung während der Tätigkeit unter Tage; radiologisches feststellbares Emphysem und/oder Silikose verschlechtern den Lungenfunktionsbefund zusätzlich. „Gesunde Bergleute verlieren ihre respiratorischen Reserven dreimal so stark wie die Allgemeinbevölkerung“ [14].

Nemery u. Mitarb. [49] beobachteten im Vergleich zu Stahlarbeitern unter nicht rauchenden belgischen Bergleuten mit und ohne Silikose ebenfalls eine Verminderung von VC, $\mathrm{FEV}_{1} \% \mathrm{VC}, \mathrm{FEV}_{1}$ und RV (die letzten beiden signifikant).

Die vorgenannten Studienergebnisse sind konsistent mit Untersuchungsdaten im britischen Steinkohlenbergbau $[40,41,65]$. Marine u. Mitarb. [41] verwendeten als Zielgrößen $\mathrm{FEV}_{1}<80 \%$, chronische Bronchitis (WHO) und zugleich $\mathrm{FEV}_{1}<80 \%$ bzw. chronische Bronchitis (WHO) und zugleich $\mathrm{FEV}_{1}<65 \%$ (der $\mathrm{FEV}_{1}$-Sollwert entsprach dabei dem Mittelwert eines internen Kontrollkollektivs). Dabei fanden sie für alle diese Zielgrößen signifikante positive Assoziation mit der kumulierten alveolengängigen Kohlengrubenstaub-Exposition und zwar unabhängig von Alter, Körpergröße und vom Rauchen; die $\mathrm{FEV}_{1}$ nahm pro $\mathrm{ghm}^{-3}$ kumulierter alveolengängiger Staubbelastung um ca. $1 \mathrm{~mL}$ ab (Nieraucher mit radiologischer Kategorie <0/1: $-1,06 \mathrm{~mL} / \mathrm{ghm}^{-3}$; Nichtraucher: $\quad-0,94 \mathrm{~mL} / \mathrm{ghm}^{-3}$; Raucher: $-1,02 \mathrm{~mL} / \mathrm{ghm}^{-3}$ ). [Beispiel für eine 20 -jährige Tätigkeit bei Grenzwertbedingungen: $0,004 \mathrm{gm}^{-3} \times 1700 \mathrm{~h} \times 20=136 \mathrm{ghm}^{-3}$ ]. Die Prävalenz einer $\mathrm{FEV}_{1}$-Einschränkung $(<80 \%$ des Sollmittelwertes) in Verbindung mit einer chronischen Bronchitis betrug unter Nichtrauchern 1,2\%. Es zeigte sich ein Anstieg der Prävalenz um 50 bzw. 100\% bei durchschnittlichen Konzentrationen des alveolengängigen Kohlengrubenstaubs von 1,2 bzw. 2,0 $\mathrm{mg} / \mathrm{m}^{3}$ im Alter von 55 Jahren nach 35-jähriger Staubexposition mit 1700 Arbeitsstunden pro Jahr [41,44].

Collins u. Mitarb. [15] beschrieben den Zusammenhang zwischen Lungenfunktionsdaten $\left(\mathrm{FEV}_{1}, \mathrm{FVC}\right)$ einerseits und der kumulierten Belastung durch alveolengängigen Staub im Steinkohlenbergbau unter a) Nierauchern ohne Silikose, mit rundlichen Lungenschatten oder unregelmäßigen Lungenschatten bzw. b) Rauchern mit kleinen unregelmäßigen Lungenschatten andererseits (Abb.5). In der multiplen Regression ergibt sich demnach im Mittel insgesamt pro ghm ${ }^{-3}$ kumulativer Exposition gegenüber alveolengängigem Staub (in Klammern: Daten für Bergleute mit unregelmäßigen Lungenschatten) ein Verlust der $\mathrm{FEV}_{1}$ von - 1,56 mL (- 1,92 mL), der FVC von - 1,51 mL (- 1,91 mL) und der $\mathrm{FEV}_{1} \% \mathrm{VC}$ von $-0,012 \%(-0,019 \%)$.

Unter nordamerikanischen Steinkohlenbergleuten waren gesteigerte Lungenfunktionsabnahmen ebenfalls überhäufig [1]. Der Rückgang in den Lungenfunktionsgrößen mit der kumulierten Exposition gegenüber alveolengängigem Staub (standardisiert nach Körpergröße, Alter, Raucherstatus und einem Indikator der jeweiligen Bergbauregion) betrug für die $\mathrm{FEV}_{1}-0,69 \mathrm{~mL} / \mathrm{ghm}^{-3}$, für die FVC $-0,49 \mathrm{~mL} / \mathrm{ghm}^{-3}$ und für die $\mathrm{FEV}_{1} \% \mathrm{FVC}$ $-8 \times 10^{-5} / \mathrm{ghm}^{-3}$ ( $\mathrm{p}$ jeweils $<0,05$ ). In der Untergruppe mit der Kategorie $0 / 0$ [32] lag die $\mathrm{FEV}_{1}$-Veränderung bei $-0,75 \mathrm{~mL} /$ $\mathrm{ghm}^{-3}$. Größere Lungenfunktionsverluste fanden sich bei höhe- 
Tab. 2a Lungenfunktionsbefunde von 50- bis 60-jährigen Bergleuten aus dem Ruhr-Steinkohlenbergbau mit und ohne Silikose sowie von einem Kontrollkollektiv, das nicht im Bergbau tätig war [63]

Angegeben sind jeweils die Mittelwerte. Berücksichtigt man die Streuung der Lungenfunktionsparameter in den einzelnen Kollektiven, so ergibt sich, dass die Werte von Bergleuten ohne Pneumokoniose deutlich überhäufig außerhalb des Bereichs der Kontrollen liegen und damit als pathologisch anzusehen sind. Si = Silikose; es sind die ILO-Klassifikationen von 1971 und 1980 [13] angegeben

\begin{tabular}{|c|c|c|c|c|c|c|}
\hline \multirow[t]{3}{*}{ Parameter } & \multicolumn{6}{|c|}{ Röntgenklassifikation } \\
\hline & \multirow{2}{*}{$\begin{array}{l}\text { Kontrollen } \\
0\end{array}$} & \multicolumn{5}{|l|}{ Bergleute } \\
\hline & & 0 & $\begin{array}{l}\text { Si I (ILO 1971) } \\
\text { (Kat. 1/0-1/2, } \\
\text { ILO 1980) }\end{array}$ & $\begin{array}{l}\text { Si II (ILO 1971) } \\
\text { (Kat. 2/1-2/3, } \\
\text { ILO 1980) }\end{array}$ & $\begin{array}{l}\text { Si III (ILO 1971) } \\
\text { (Kat. } \geq 3 / 2, \\
\text { ILO 1980) }\end{array}$ & $\begin{array}{l}\text { Pinhead } \\
\text { (<1,5 mm, } \\
\text { submiliare Si) }\end{array}$ \\
\hline$V C(m L)$ & 4150 & 3560 & 3550 & 3370 & 3430 & 3100 \\
\hline$R V(m L)$ & 1920 & 2260 & 2450 & 2360 & 2220 & 2530 \\
\hline$R_{t}\left(\mathrm{kPa} \cdot \mathrm{L}^{-1} \cdot \mathrm{s}\right)$ & 0,218 & 0,212 & 0,213 & 0,209 & 0,211 & 0,219 \\
\hline$V_{D} / V_{T}$ & 0,31 & 0,38 & 0,38 & 0,39 & 0,38 & 0,39 \\
\hline$V_{D, O_{2}}$ & 162 & 201 & 218 & 241 & 244 & 232 \\
\hline$P_{(A-a), O_{2}}(m m H g)$ & 15,3 & 27,8 & 26,5 & 28,7 & 26,6 & 31,9 \\
\hline
\end{tabular}

Tab. 2b Lungenfunktionsbefunde von 50- bis 60-jährigen Bergleuten aus dem Ruhr-Steinkohlenbergbau mit und ohne Silikose sowie von einem Kontrollkollektiv, das nicht im Bergbau tätig war (zitiert nach Reichel [53])

Angegeben sind jeweils die Mittelwerte. Berücksichtigt man die Streuung der Lungenfunktionsparameter in den einzelnen Kollektiven, so ergibt sich, dass die Werte von Bergleuten ohne Pneumokoniose deutlich überhäufig außerhalb des Bereichs der Kontrollen liegen und damit als pathologisch anzusehen sind

\begin{tabular}{|c|c|c|c|c|}
\hline \multirow[t]{3}{*}{ Parameter } & \multicolumn{4}{|c|}{ Röntgenklassifikation (ILO 1971) } \\
\hline & Kontrollen & Bergleute & & \\
\hline & 0 & 0 & Apqr & $B C$ \\
\hline$V C(m L)$ & 3800 & 3400 & 3200 & 3300 \\
\hline$F E V_{1}(m L)$ & 2800 & 2500 & 2450 & 2200 \\
\hline$R_{t}\left(\mathrm{kPa} \cdot \mathrm{L}^{-1} \cdot \mathrm{s}\right)$ & 0,305 & 0,395 & 0,40 & 0,53 \\
\hline
\end{tabular}

Tab. 2c Vitalkapazität (VC) von Steinkohlenbergleuten verschiedener Altersgruppen im Vergleich zu gleichaltrigen nicht exponierten Kontrollen [14]

\begin{tabular}{|lllll}
\hline \multicolumn{2}{l}{ VC in \% des Sollmittelwertes } & & & \\
\hline & bis 29 J. & $30-39 J$. & $40-49 J$. & 92 \\
\hline Kontrollen & 100 & 97 & 92 & 88 \\
\hline gesunde Bergleute & 116 & 107 & 97 & 79 \\
\hline Bergleute m. Bronchitis & 114 & 104 & 81 & 75 \\
\hline Bergleute m. Silikose & - & 97 & 78 & 73 \\
\hline
\end{tabular}

rem Inkohlungsgrad (adjustiert nach der kumulierten alveolengängigen Kohlengrubenstaubexposition).

Seixas u. Mitarb. ([61,62] Abb. 2 a u. b) konnten eine signifikante Zunahme der Prävalenz einer eingeschränkten Lungenfunktion $\left(\mathrm{FEV}_{1}<80 \%, \mathrm{FEV}_{1} \% \mathrm{FVC}<80 \%\right.$ des Sollmittelwertes nach Crapo u. Mitarb. [16], von chronischem Auswurf, chronischer Bronchitis und Atemnot in Abhängigkeit von der jeweils kumulierten Staubexposition feststellen. Dabei zeigten sich hohe Verluste in den Lungenfunktionswerten vornehmlich in den ersten Jahren nach Beginn der untertägigen Tätigkeit, aber auch Langzeiteffekte der Kohlengrubenfeinstaub-Exposition.

Von Leigh (1990) wurde unter australischen Kohlenbergarbeitern in einem wiederholten Querschnitt ein $\mathrm{FEV}_{1}$-Abfall von $810 \mathrm{~mL}$ in 15 Tätigkeitsjahren beschrieben (erwartete altersbedingte Änderung: $300 \mathrm{~mL}$ ). 

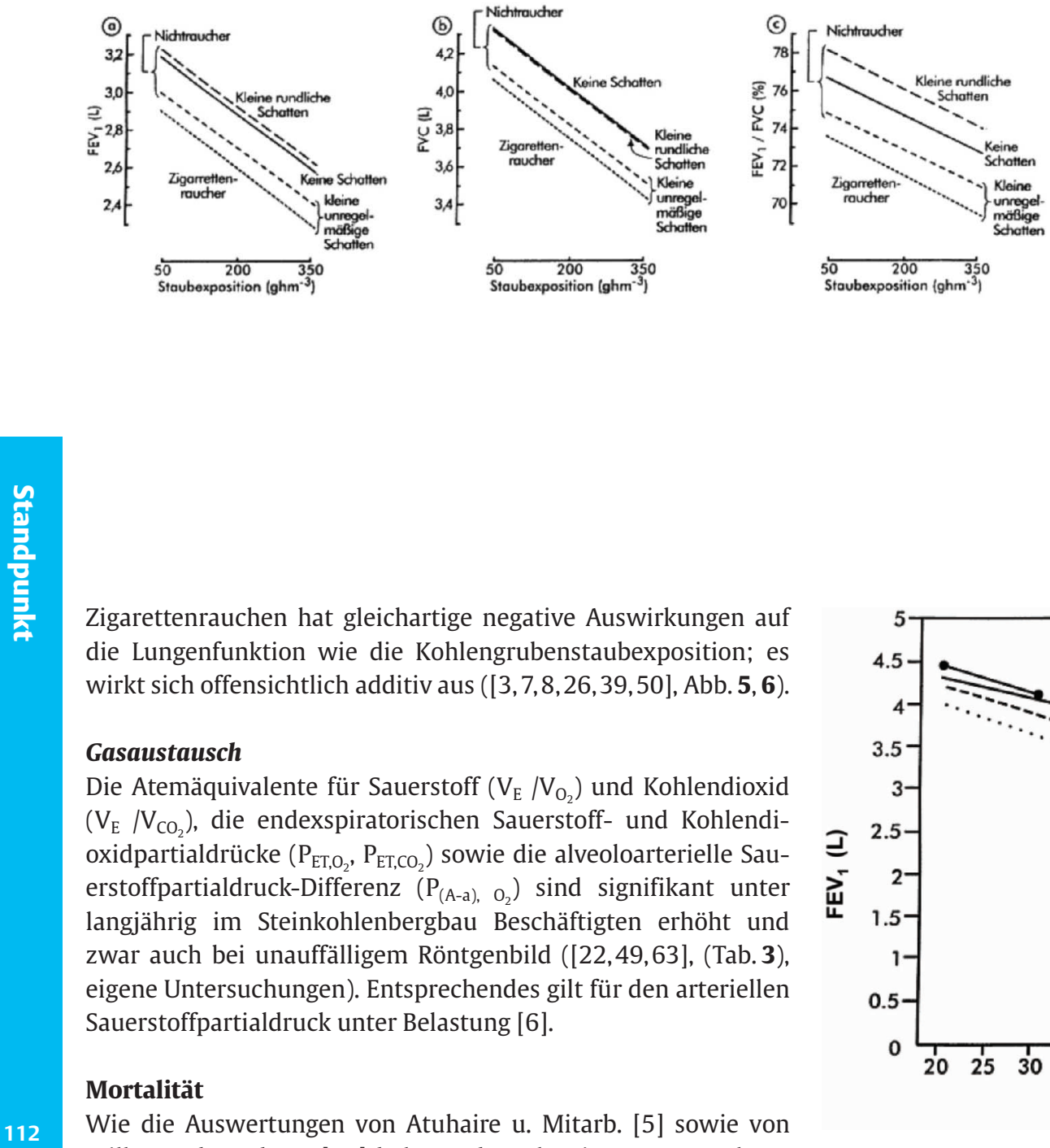

Zigarettenrauchen hat gleichartige negative Auswirkungen auf die Lungenfunktion wie die Kohlengrubenstaubexposition; es wirkt sich offensichtlich additiv aus ([3, 7,8,26,39,50], Abb. 5, 6).

\section{Gasaustausch}

Die Atemäquivalente für Sauerstoff $\left(\mathrm{V}_{\mathrm{E}} / \mathrm{V}_{\mathrm{O}_{2}}\right)$ und Kohlendioxid $\left(\mathrm{V}_{\mathrm{E}} / \mathrm{V}_{\mathrm{CO}_{2}}\right)$, die endexspiratorischen Sauerstoff- und Kohlendioxidpartialdrücke $\left(\mathrm{P}_{\mathrm{ET}, \mathrm{O}_{2}}, \mathrm{P}_{\mathrm{ET}, \mathrm{CO}_{2}}\right)$ sowie die alveoloarterielle Sauerstoffpartialdruck-Differenz $\left(\mathrm{P}_{(\mathrm{A}-\mathrm{a}), \mathrm{O}_{2}}\right)$ sind signifikant unter langjährig im Steinkohlenbergbau Beschäftigten erhöht und zwar auch bei unauffälligem Röntgenbild ([22,49,63], (Tab.3), eigene Untersuchungen). Entsprechendes gilt für den arteriellen Sauerstoffpartialdruck unter Belastung [6].

\section{Mortalität}

Wie die Auswertungen von Atuhaire u. Mitarb. [5] sowie von Miller und Jacobsen [43] belegen, besteht ein Zusammenhang zwischen der geschätzten individuellen kumulativen Staubdosis des Steinkohlenbergmanns bzw. der radiologisch festgestellten Silikose-Kategorie einerseits und der Todesursache „Pneumokoniose“ andererseits. Eine signifikant erhöhte Mortalität aufgrund von Pneumokoniose wurde auch für nordamerikanische Steinkohlenbergleute beschrieben (SMR für Pneumokoniose 9,3 bzw. 3,72 [57]).

Vallyathan u. Mitarb. [71, 72] und Attfield u. Mitarb. [4] werteten Obduktionsbefunde von US-amerikanischen Steinkohlenbergleuten aus. Sie beobachteten eine Abhängigkeit des Schweregrades eines Lungenemphysems von den Rauchgewohnheiten, der Dauer der Untertagetätigkeit, der Gesamt- und Kohlenstaubmenge in der Lunge und dem pathologisch-anatomisch objektivierten Pneumokoniose-Grad. Vergleichbare Befunde erhoben Leigh u. Mitarb. [38] unter australischen Steinkohlenbergleuten. Dagegen fand sich in diesen Studien keine Assoziation des Lungenemphysems mit der inkorporierten Mineral- und Quarzstaubmenge, vielmehr war ein Zusammenhang zwischen dem pathologisch verifizierten Fibrosegrad und dem Quarzgehalt der Lunge festzustellen.

Höhergradige pathologisch-anatomische Lungenbefunde waren - wie Vallyathan u. Mitarb. [71,72]. mitteilen - mit röntgenologisch feststellbarer Pneumokoniose vergesellschaftet, nicht jedoch die geringgradigen Obduktionsbefunde. $22 \%$ der patholo-
Abb. 5 Multiple Regression über den Zusammenhang zwischen Lungenfunktion $\left(\mathrm{FEV}_{1}, \mathrm{FVC}\right)$ und der kumulativen Staubbelastung im Steinkohlenbergbau unter Nichtrauchern ohne Silikose (-), Nichtrauchern mit kleinen runden Lungenschatten (---), kleinen unregelmäßigen Lungenschatten (---), ferner von Zigarettenrauchern mit kleinen unregelmäßigen Lungenschatten (….) [15]. Es sind die Daten eines 49-jährigen Bergmanns (Körpergröße $170 \mathrm{~cm}$, Gewicht $74 \mathrm{~kg}$ ) dargestellt. Für $100 \mathrm{ghm}^{-3} \mathrm{ku}-$ mulativer Feinstaubexposition ergibt sich im Mittel insgesamt (in Klammern: Daten der Bergleute mit unregelmäßigen Lungenschatten) ein Verlust der $\mathrm{FEV}_{1}$ von $-156 \mathrm{~mL}$ $(-192 \mathrm{~mL})$, der FVC von $-151 \mathrm{~mL}$ $(-191 \mathrm{~mL})$ und der FEV ${ }_{1} \% \mathrm{FVC}$ von $-1,2 \%$ $(-1,9 \%)$.

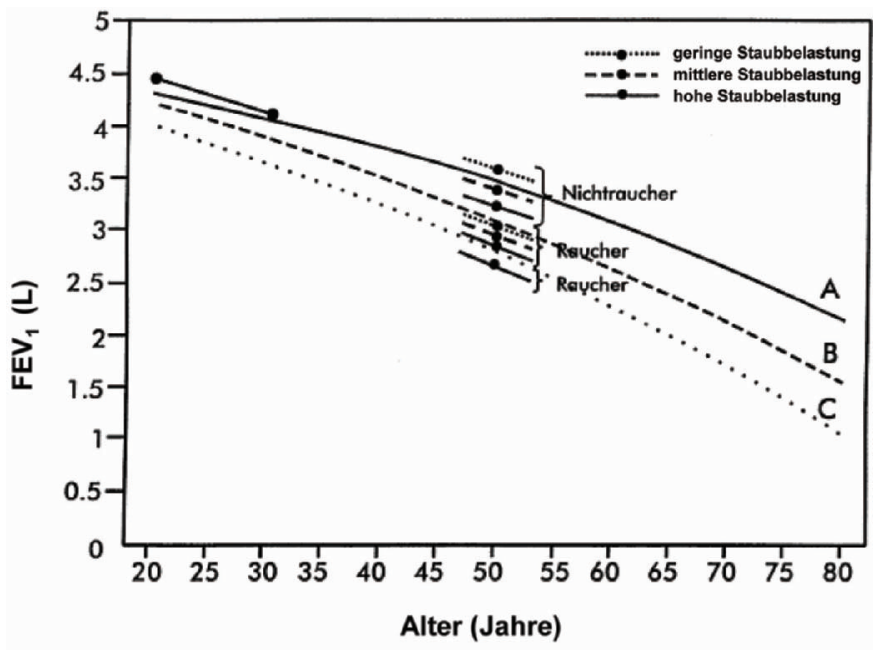

Abb. 6 FEV 1 -Werte der Goldminenarbeiter in Abhängigkeit von der Höhe der stattgehabten Staubexposition und dem Rauchverhalten. Die Daten sind über die Vorhersagewerte von nicht exponierten Nichtrauchern (A), Rauchern mit 1 Packung Zigaretten pro Tag (B) bzw. mit 2 Packungen Zigaretten pro Tag (C) projektiert [26]. Links oben (--.): $\mathrm{FEV}_{1}$-Werte von 20-30-jährigen Bergleuten (Raucher und Nichtraucher; vgl. Healthy Worker Effect).

Tab. 3 Spiroergometrische Befunde von Bergleuten aus dem RuhrSteinkohlenbergbau mit Silikose der Kategorie 1 und 2 sowie eines Kontrollkollektivs, das nicht im Bergbau tätig war [22].

Für sämtliche Parameter liegen signifikante Unterschiede zwischen beiden Gruppen vor

\begin{tabular}{lll}
\hline Parameter & Kontrollen & Bergleute \\
\hline$\left(V_{E} / V_{\mathrm{O}_{2}}\right)$ & 25,71 & 32,90 \\
\hline$\left(V_{E} V_{\mathrm{CO}_{2}}\right)$ & 30,98 & 39,40 \\
\hline$P_{E T, \mathrm{O}_{2}}(\mathrm{~mm} \mathrm{Hg})$ & 99,08 & 115,60 \\
\hline$P_{E T, \mathrm{O}_{2}}(\mathrm{~mm} \mathrm{Hg})$ & 44,04 & 38,38 \\
\hline
\end{tabular}


Tab. 4 Sektionsbefunde von britischen Steinkohlenbergleuten: Anteil der Lungen mit Emphysem in Prozent (Anzahl) in Abhängigkeit von der Art der radiologisch festgestellten Lungenschatten [58]

\begin{tabular}{lllll|}
\hline \multicolumn{5}{c}{ röntgenologisch festgestellte Lungenschatten } \\
\hline $\begin{array}{l}\text { Sektionsbefund } \\
\text { nicht schwielige }\end{array}$ & $\begin{array}{l}\text { keine Schatten } \\
\text { Silikose }\end{array}$ & nur p, q, r & p, q, r \& s, t, u nur s, t, u \\
$\begin{array}{l}\text { alle Bergleute (inkl. } \\
\text { nicht schwielige }\end{array}$ & $57 \%(53)$ & $87 \%(23)$ & $92 \%(13)$ \\
\begin{tabular}{l} 
Silikose) \\
\hline
\end{tabular} & $64 \%(96)$ & $89 \%(79)$ & $92 \%(24)$ \\
\hline
\end{tabular}

Tab. 5 Sektionsbefunde von britischen Steinkohlenbergleuten: Anteil der Lungen mit Emphysem in Prozent (Anzahl) in Abhängigkeit von der Größe der röntgenologisch festgestellten kleinen runden Lungenschatten [58]

röntgenologisch festgestellte Lungenschatten

\begin{tabular}{llll}
\hline Sektionsbefund & $\mathrm{p}(<1,5 \mathrm{~mm})$ & $\mathrm{q}(1,5-3 \mathrm{~mm})$ & $\mathrm{r}(3-10 \mathrm{~mm})$ \\
$\begin{array}{l}\text { nicht schwielige } \\
\text { Silikose }\end{array}$ & $89 \%(18)$ & $61 \%(41)$ & $(2)^{\mathrm{a}}$ \\
$\begin{array}{l}\text { alle Bergleute (inkl. } \\
\text { nicht schwielige }\end{array}$ & $92 \%(37)$ & $66 \%(80)$ & $57 \%(14)$ \\
\begin{tabular}{l} 
Silikose) \\
\hline
\end{tabular} & & \\
\hline
\end{tabular}

aNur 2 Männer mit „r“-Lungenschatten hatten keine nicht-schwielige Silikose; einer davon hatte ein Emphysem

gisch gesicherten Schwielen waren radiologisch nicht entdeckt worden. Andererseits waren 25\% der Fälle mit röntgenologisch diagnostizierter Silikose nicht mit einem pathologischen Korrelat vergesellschaftet, sondern Fehldiagnosen, z. B. Narbenbildungen infolge einer Tuberkulose oder Tumoren. Ähnliche Diskrepanzen fanden Hnizdo [26] und Hnizdo u. Mitarb. [27] unter Goldminenarbeitern.

Ruckley u. Mitarb. [58,59] erhoben Sektionsbefunde von verstorbenen britischen Steinkohlenbergleuten. Dabei zeigte sich hier noch deutlicher die geringe Sensitivität konventioneller Röntgenuntersuchungen: Die Hälfte der Untersuchten mit unauffälligem Thoraxbild und ohne röntgenologische Zeichen einer Silikose hatte ein Lungenemphysem. Am häufigsten waren Lungenemphyseme unter Steinkohlenbergleuten mit kleinen rundlichen Lungenschatten bis 1,5 mm Durchmesser anzutreffen. Es wurde eine Assoziation sowohl zwischen diesen p-Schatten als auch kleinen unregelmäßigen Lungenverschattungen $(s, t, u)$ einerseits und dem Vorliegen eines Lungenemphysems beobachtet (Tab. 4 u. 5).

\section{Lungenkrebs}

Kohlenbergleute können sowohl gegenüber Quarzstaub aus dem Nebengestein als auch gegenüber dem Quarzanteil (5-12\%) im Kohlengrubenstaub exponiert sein. Alveolengängiger Quarzstaub wurde von der „International Agency for Research on Can- cer" [31] und der Senatskommission zur Prüfung gesundheitsschädlicher Arbeitsstoffe der Deutschen Forschungsgemeinschaft [21] als krebserzeugende Substanz beim Menschen (Kategorie 1 der krebserzeugenden Arbeitsstoffe) eingestuft. Dabei ist das Lungenkrebsrisiko mindestens verdoppelt, wenn eine Silikose vorliegt $[24,64,67]$. Dagegen sind die Evidenzen für eine Kanzerogenität von Kohlengrubenstaub unzureichend [21,31]. Während ein erhöhtes Lungenkrebsrisiko bei Vorliegen einer Silikose außerhalb des Kohlenbergbaus wissenschaftlich gesichert ist, ist der Zusammenhang zwischen Lungenkrebs und einer Pneumokoniose des Bergmanns entweder nicht gegeben oder wissenschaftlich nur schwer nachweisbar [37]. Bemerkenswert sind konsistente Befunde bez. eines erhöhten Risikos eines Magenkrebses der Steinkohlenbergleute; eine Dosis-Wirkungs-Beziehung zwischen der Krebshäufigkeit und der Expositionshöhe bzw. Expositionszeit konnte jedoch bisher nicht eindeutig nachgewiesen werden.

\section{Diskussion}

Der Zusammenhang zwischen der Untertagetätigkeit im Steinkohlenbergbau und dem Auftreten von chronischer Bronchitis, obstruktiver Atemwegserkrankung und Lungenemphysem mit oder ohne radiologisch fassbare Pneumokoniose (Silikose) wurde konsistent in großen Studien belegt (Übersichten bei $[8,18-21,41,44,50,73]$. Dabei wurden wegen des nicht berücksichtigten überdurchschnittlich guten Gesundheitszustandes der Berufsanfänger sowie des überhäufigen Ausscheidens von Beschwerdeträgern (Healthy-Worker-Effekte) die adversen Auswirkungen unterschätzt $[27,30,73]$.

Die Trennung von Silikose und chronischer obstruktiver Bronchitis/Emphysem (CB-E) im Berufskrankheitenrecht Deutschlands ist historisch bedingt und letztendlich artifiziell. Pathogenetisch, pathophysiologisch und funktionell gesehen liegt bei beiden Berufskrankheitsdiagnosen dasselbe Geschehen zugrunde. Lediglich der Grad der radiologisch darstellbaren Fibrose des Lungengewebes ist verschieden. Charakteristisch ist die im Mittel leichtgradige Restriktion, die nach 20-jähriger und längerer Tätigkeit bei ca. $1 / 4$ der Steinkohlenbergleute mit pathologischen Lungenfunktionswerten einhergeht. Neben dieser restriktiven Komponente sind unter langjährig im Steinkohlenbergbau Tätigen überhäufig eine chronische obstruktive Bronchitis und ein Lungenemphysem anzutreffen. Diese relativ häufigen klinisch manifesten Spätkomplikationen der Kohlengrubenstaub-Inkorporation, das Lungenemphysem und die chronische obstruktive Bronchitis, können individuell stark unterschiedlich ausgeprägt sein. Stärker belastete Bergleute zeigen dabei deutlichere Effekte als gering oder mäßig belastete. Ein Extrem stellt die „Schwarze Löcherlunge“ dar, die sich durch ein schweres, vorwiegend zentrilobuläres Lungenemphysem auszeichnet [46].

Versicherungsrechtlich und gutachterlich sind neben dem aktuellen wissenschaftlichen Kenntnisstand, die im Einzelfall mittels moderner, sensitiver Verfahren qualitätsgesichert erhobenen Untersuchungsparameter zu berücksichtigen. Es sollte nicht vorkommen, dass allein anhand des Röntgenbefundes eine völlig unterschiedliche Fallbewertung, letztlich Ablehnung oder Anerkennung einer Berufskrankheit, resultiert. Vielmehr müssen 
grundsätzlich eine Gesamtschau der klinischen Befunde und des Krankheitsverlaufs sowie eine unvoreingenommene Zusammenhangsbeurteilung erfolgen. Da die Funktionsausfälle großteils unabhängig vom radiologischen Befund sind, ist es nicht begründbar, bei Silikosekranken mit einer Kategorie von $<^{2} / 3$ (ILO 1980) [32] einen kausalen Zusammenhang zwischen der beruflichen Belastung und dem Krankheitsbild grundsätzlich auszuschließen [9-11,66,70,74]. Vielmehr ist der vorliegende Gesundheitsschaden wie in jedem anderen Berufskrankheits-Verdachtsfall individuell ätiologisch zu ermitteln und mit Wahrscheinlichkeit zuzuordnen, d. h. auch andere Krankheitsursachen sind zu eruieren, zu bewerten und abzugrenzen. Nur wenn nichtberufliche Ursachen zweifelsfrei ganz überwiegend das Krankheitsgeschehen hervorrufen, wird bei gegebener haftungsbegründender Kausalität (=langjährige Untertagetätigkeit im Steinkohlenbergbau) ein Berufskrankheits-Entschädigungsfall zu verneinen sein.

Die Berufskrankheits-Anerkennungspraxis bezüglich des Bronchialkarzinoms des Steinkohlen-Bergmanns, die sich bisher an der Nachbarschaft einer (zufällig) gefundenen Narbe orientiert, ist unbefriedigend. Epidemiologische Studien belegen klar den Zusammenhang zwischen der Exposition gegenüber alveolengängigem kristallinem Siliciumdioxid (v.a. Quarz) und der Silikoseerkrankung, der chronischen obstruktiven Bronchitis, dem Lungenemphysem sowie dem Bronchialkarzinom (letzteres bisher nur außerhalb des Steinkohlenbergbaus; warum für den Steinkohlenbergbau ein solcher Zusammenhang bisher nicht nachgewiesen werden konnte, ist unklar und bedarf einer Überprüfung; [37]).

\section{Literatur}

${ }^{1}$ Attfield MD, Hodous TK. Pulmonary function of U. S. coal miners related to dust exposure estimates. Am Rev Respir Dis 1992; 145: 605-609

${ }^{2}$ Attfield MD, Morring K. An investigation into the relationship between coal workers pneumoconiosis and dust exposure in U. S. coal miners. Am Ind Hyg Assoc J 1992; 53: 486 - 492

${ }^{3}$ Attfield MD. Longitudinal decline in $\mathrm{FEV}_{1}$ in United States coalminers. Thorax 1985; 40: $132-137$

${ }^{4}$ Attfield MD, Vallyathan V, Green FHY. Radiographic appearances of small opacities and their correlation with pathology grading of macules, nodules and dust burden in the lungs. Ann Occup Hyg 1994; 38: $782-789$

${ }^{5}$ Atuhaire LK, Campbell MJ, Cochrane AL et al. Mortality of men in the Rhondda Fach 1950 - 1980. Br J Ind Med 1985; 42: 741 - 745

${ }^{6}$ Bauer TT. Korrelation zwischen röntgenmorphologischen und funktionsanalytischen Befunden bei Quarz- und Kohlenstaub-bedingten Atemwegs- und Lungenkrankheiten (Anthrakosilikose). Abschlussbericht (Teil 2) und Zusammenfassung der Untersuchungsergebnisse. Bochum: Berufsgenossenschaftliche Kliniken Bergmannsheil. Abteilung für Pneumologie, Allergologie und Schlafmedizin, 2003

${ }^{7}$ Baur X, Hillenbach C, Degens P. Literaturstudie: Chronische Bronchitis und Emphysem - eine Berufskrankheit der Bergleute? Berufsgenossenschaftliches Forschungsinstitut für Arbeitsmedizin. Bochum: Eigenverlag, 1994

8 Baur X, Degens P. Chronische Bronchitis und Emphysem - eine Berufskrankheit der Bergleute? Tagung der Arbeitsgemeinschaft des Saarlandes zur Erforschung und Förderung des Gesundheitsschutzes im Bergbau e. V. (AGiB). Saarbrücken: Neunkirchener Druckerei und Verlag, 1994: 57-61

${ }^{9}$ Baur X, Degens P. Minderung der Erwerbsfähigkeit bei gering gestreuten Silikosen der Steinkohlenbergleute - Ist eine Neubewertung der
„Moerser Konvention“ erforderlich? Atemwegs-Lungenkrkh 2000; 26: $371-376$

${ }^{10}$ Baur X. Silikose und MdE. In: Landesverband Rheinland-Westfalen (Hrsg). 16. Duisburger Gutachter-Kolloquium Berufskrankheiten-Teil. Dezember 1999. Düsseldorf: Verlag gewerblicher Berufsgenossenschaften, 2001: 110 - 129

${ }^{11}$ Baur X. Zur Begutachtung der Silikose (BK 4101) sowie der chronischen obstruktiven Bronchitis und des Emphysems der Steinkohlenbergleute (BK 4111). Arbeitsmed, Sozialmed, Umweltmed 1999; 34: $368-370$

12 Becklake MR. The mineral dust diseases. Tuber Lung Dis 1992; 73: $13-20$

${ }^{13}$ Bohlig H, Hain E, Valentin $\mathrm{H}$ et al. Die Weiterentwicklung der Internationalen Staublungenklassifikation und ihre Konsequenzen für die arbeitsmedizinischen Vorsorgeuntersuchungen staubgefährdeter $\mathrm{Ar}$ beitnehmer (ILO 1980/Bundesrepublik). Prax Pneumol 1981; 35: $1134-1139$

${ }^{14}$ Carstens M, Brinkmann O, Lange HJ et al. Beiträge zur Pathophysiologie der Staublungenkrankheit im Bergbau. III. Mitteilung. Archiv Gewerbepath Gewerbehyg 1958; 16: 459-477

${ }^{15}$ Collins HP, Jacobsen M, Dick JA et al. Irregularly shaped small shadows on chest radiographs, dust exposure, and lung function in coalworkers' pneumoconiosis. Br J Ind Med 1988; 45: 43-55

${ }^{16}$ Crapo RO, Morris AH, Gardner RM. Reference spirometric values for spirometry using techniques and equipment that meets ATS recommendations. Am Rev Respir Dis 1981; 123: 659-664

17 Degens P, Baur X. Besteht heute noch ein Risiko bezüglich einer Silikose-Erkrankung? Pneumologie 1994; 48: 513 - 515

18 Deutsche Forschungsgemeinschaft (DFG). Quarz-Feinstaub und quarzhaltiger Feinstaub. In: Henschler D (Hrsg). Gesundheitsschädliche Arbeitsstoffe, Toxikologisch-Arbeitsmedizinische Begründung von MAK-Werten. Weinheim: Chemie Verlag, 1971: 121

${ }^{19}$ Deutsche Forschungsgemeinschaft (DFG). Quarz-Feinstaub und quarzhaltiger Feinstaub - Nachtrag. In: Henschler D (Hrsg). Gesundheitsschädliche Arbeitsstoffe, Toxikologisch-Arbeitsmedizinische Begründung von MAK-Werten. Weinheim: Chemie Verlag, 1984: 1-8

${ }^{20}$ Deutsche Forschungsgemeinschaft (DFG). Arbeitsmedizinische Querschnittsuntersuchungen zur Bedeutung chronischer inhalativer Belastungen für das bronchopulmonale System. Forschungsbericht chronische Bronchitis und Staubbelastung am Arbeitsplatz. Boppard: Harald Bolt Verlag, 1975

${ }^{21}$ Deutsche Forschungsgemeinschaft (DFG). Steinkohlengrubenstaub. In: Greim H (Hrsg). Gesundheitsschädliche Arbeitsstoffe. Toxikologisch-arbeitsmedizinische Begründungen von MAK-Werten. Senatskommission zur Prüfung gesundheitsschädlicher Arbeitsstoffe. Weinheim: VCH Verlagsgesellschaft, 1998: 1-51

22 Duvenkamp I, Bauer TT, Schmidt EW et al. Submaximale spiroergometrische Belastungsuntersuchung bei Patienten mit Mischstaub-Pneumokoniosen. Pneumologie 1998; 52: 171 - 177

${ }^{23}$ Finkelstein MM. Silicosis Surveillance in Ontario: detection rates, modifying factors, and screening intervals. Am J Ind Med 1994; 25: 257-266

${ }^{24}$ Heuchert G. Bewertung epidemiologischer Studien zur Analyse der Beziehungen zwischen silikose-induzierenden Feinstaubexpositionen und Lungenkrebs. Bundesanstalt für Arbeitsschutz und Arbeitsmedizin Dortmund, Berlin (Hrsg). Bremerhaven: Wirtschaftsverlag N. W., 1999. (Schriftenreihe der Bundesanstalt für Arbeitsschutz und $\mathrm{Ar}$ beitsmedizin; Sonderschrift S57), 1999

${ }^{25}$ Hnizdo E, Sluis-Cremer GK. Risk of silicosis in a cohort of White South African gold miners. Am J Ind Med 1993; 24: 447-457

${ }^{26}$ Hnizdo E. Health risks among white South African gold miners - dust, smoking and chronic obstructive pulmonary disease. S Afr Med J 1992; 81: $512-517$

${ }^{27}$ Hnizdo E, Murray J, Sluis-Cremer GK et al. Correlation between radiological and pathological diagnosis of silicosis: An autopsy population based study. Am J Ind Med 1993; 24: 427-445

${ }^{28}$ Hurley JF, Maclaren WM. Dust-related risks of radiological changes in coal miners over a 40-year working life: report on work commissioned by NIOSH. Edinburgh: Institute of Occupational Medicine (Report No. TM/87/091), 1987

${ }^{29}$ Hurley JF, Burns J, Copland L et al. Coalworkers simple pneumoconiosis and exposure to dust at 10 British coalmines. Br J Ind Med 1982; 39: $120-127$

${ }^{30}$ Hurley JF, Soutar CA. Can exposure to coalmine dust cause a severe impairment of lung function? $\mathrm{Br} \mathrm{J}$ Ind Med 1986; 43: 150-157 
${ }^{31}$ International Agency for Research on Cancer (IARC). Silica, some silicates, coal dust and para-aramid fibrils. IARC monographs on the evaluation of carcinogenic risks to humans 1997; 68: 337-406

32 International Labour Office (ILO). Guidelines for the use of ILO International Classification of Radiographs of Pneumoconiosis. Revised edition 1980. Geneva: ILO (Occupational Safety and Health Series 22), 1980

${ }^{33}$ International Labour Office (ILO). International Classification of Radiographs of Pneumocosiosis (revised 1968). Geneva: ILO (Occupational Safety and Health Series 22), 1970

${ }^{34}$ International Labour Office (ILO). Meeting of experts on the International Classification of Radiographs of the Pneumoconiosis. Geneva: ILO (Occupational Safety and Health Series 2), 1959

35 Jacobsen M, Burns J, Attfield AD. Sending and coalworkers' simple pneumoconiosis. In: Walton WH, McGovern B (Hrsg). Inhaled particles IV. Oxford: Pergamon Press, 1977: 759- 772

${ }^{36}$ Lange HJ, Pache J. Bericht über Auswertung von Daten der KnRV der ArV und der AnV zur Frage von chronischer Bronchitis und/oder Emphysem als arbeitsbedingte Erkrankung der Bergleute. Arbeitsmed Sozialmed Präventivmed 1991; (Sonderheft 17): 3-46

${ }^{37}$ Latza U, Degens P, Baur X. Das Lungenkrebsrisiko bei beruflicher Quarz- und Kohlengrubenstaubexposition. Arbeitsmed Sozialmed Umweltmed 2000; 35: 424-438

${ }^{38}$ Leigh J, Driscoll TR, Cole BD et al. Quantitative relation between emphysema and lung mineral content in coalworkers. Occup Environ Med 1994; 51: 400-407

${ }^{39}$ Leigh J. 15 year longitudinal studies of $\mathrm{FEV}_{1}$ loss and mucus hypersecretion development in coalworkers in New South Wales, Australia. In: Proceedings of the VIIth International Pneumoconiosis Conference 1988. Pittsburgh, 1990: 112 - 121

${ }^{40}$ Love RG, Miller BG. Longitudinal study of lung function in coalminers. Thorax 1982; 37: $193-197$

41 Marine WM, Gurr D, Jacobsen M. Clinically important respiratory effects of dust exposure and smoking in British coal miners. Am Rev Respir Dis 1988; 137: 106-112

${ }^{42}$ Martin JR, Muir DC, Moore E et al. Pneumoconiosis in iron or surface mining in Labrador. J Occup Med 1988; 30: 780 - 784

${ }^{43}$ Miller BG, Jacobsen M. Dust exposure pneumoconiosis and mortality of coal miners. Br J Ind Med 1985; 42: 723 - 733

${ }^{44}$ Morfeld P, Piekarski C. Epidemiologie der Pneumokoniose und der chronischen Bronchitis im Steinkohlenbergbau. Bundesanstalt für Arbeitsschutz und Arbeitsmedizin Dortmund, Berlin (Hrsg). Bremerhaven: Wirtschaftsverlag N. W., 1998. (Schriftenreihe der Bundesanstalt für Arbeitsschutz und Arbeitsmedizin; Sonderschrift S45) 1998

45 Morfeld P, Vautrin HJ, Kösters A et al. l. Components of coal mine dust exposure and the occurance of pre-stages of pneumoconiosis. J Appl Occup Environ Hyg 1997; 12: 973 - 979

46 Müller K-M, Worth G. Ein Beitrag zum „Kohlenstaubemphysem“ („schwarze Löcherlunge“). Prax Pneumol 1978; 32: 231 - 237

47 Muhle H, Creutzenberg O, Bellmann B et al. Dust overload in lungs: investigations of various materials, species differences, and irreversibility of effects. J Aerosol Med 1990; 3 Suppl 1: $11-128$

${ }^{48}$ Murray M, Finkelstein M. Silicosis surveillance in Ontario: Detection rates, modifying factors, and screening intervals. Am J Ind Med 1994; 25: $257-266$

${ }^{49}$ Nemery B, Brasseur L, Veriter C et al. Impairment of ventilatory function on pulmonary gas exchange in non-smoking coalminers. Lancet 1987; 2 (8573): $1427-1430$

50 Oxman AD, Muir DCF, Shannon HS et al. Occupational dust exposure and chronic obstructive pulmonary disease. Am Rev Respir Dis 1993; 148: $38-48$

51 Piontek K, Hoffarth HP, Robock K et al. Einfluss der Schichthorizonte auf die Entwicklungsgeschwindigkeit der Bergarbeiter-Pneumokoniose (Silikose). Arbeitsmed Sozialmed Präventivmed 1991; 26: 89-93

52 Quanjer PH. Standardized lung function testing. Bull Eur Physiopath Respir 1983; 5: 1 - 92

${ }^{53}$ Reichel G. Die Silikose (Anthrakosilikose). In: Ulmer WT, Reichel G (Hrsg). Pneumokoniosen - Handbuch der Inneren Medizin, 5. Auflage,
Band 4 Atmungsorgane 1. Teil. Berlin, Heidelberg, New York: Springer, 1976

${ }^{54}$ Reisner MT, Robock K. Results of epidemiological mineralogical and cytotoxicological studies on the pathogenicity of coal-mine custs. Inhaled Part 1975; 2: $703-716$

${ }^{55}$ Reisner MTR, Kotitschke G, Niesert E. Pneumokoniose und Staubexposition - Epidemiologische Untersuchungen im Steinkohlenbergbau an der Ruhr über einen Zeitraum von 20 Jahren. Silikosebericht Nordrhein-Westfalen 1985; 15: 445-492

${ }^{56}$ Reisner MTR. Pneumokoniose und Staubexposition - Epidemiologische Untersuchungen im Ruhrgebiet über einen Zeitraum von 14 Jahren. Silikosebericht Nordrhein-Westfalen 1975; 10: 209-231

${ }^{57}$ Rockette HE. Cause specific mortality of coal miners. J Occup Med 1977; 19: 795-801

${ }^{58}$ Ruckley VA, Fernie JM, Chapman JS et al. Comparison of radiographic appearances with associated pathology and lung dust content in a group of coalworkers. Br J Ind Med 1984; 41: 459-467

59 Ruckley VA, Gauld SJ, Chapman JS et al. Emphysema and dust exposure in a group of coal workers. Am Rev Respir Dis 1984; 129: 528 - 532

60 Schins RPF, Borm PJA. Mechanisms and mediators in coal dust induced toxicity: a review. Ann Occup Hyg 1999; 43: 7- 33

${ }^{61}$ Seixas NS, Robins TG, Attfield MD et al. Exposure-response relationships for coal mine dust and obstructive lung disease following enactment of the Federal Coal Mine Health and Safety Act of 1969. Am J Ind Med 1992; 21: $715-734$

62 Seixas NS, Robins TG, Attfield MD et al. Longitudinal and cross sectional analyses of exposure to coal mine dust and pulmonary function in new miners. Br J Ind Med 1993; 50: 929-937

${ }^{63}$ Smidt U. Distribution of inhaled air in coal workers with and without silicosis. Rev Inst Hyg Mines (Hasselt) 1974; 29: 72 - 84

${ }^{64}$ Smith AH, Lopipero PA, Barroga VR. Meta-Analysis of studies of lung cancer among Silicotics. Epidemiology 1995; 6: 617-624

${ }^{65}$ Soutar CA, Hurley JF. Relation between dust exposure and lung function in miners and ex-miners. Br J Ind Med 1986; 43: 307-320

${ }^{66}$ Steger A, Pethran A, Fruhmann G. Aspekte medizinischer Fachgutachten für Sozialgerichte mit besonderer Berücksichtigung der Berufskrankheiten Silikose und Siliko-Tuberkulose. Arbeitsmed Sozialmed Umweltmed 1998; 33: $381-387$

67 Tsuda T, Babazono A, Yamamoto E et al. A meta-analysis on the relationship between pneumoconiosis and lung cancer. J Occup Health 1997; 39: $285-294$

68 Ulmer WT, Reichel G, Roeske G et al. Klinische und funktionsanalytische Untersuchungen bei Bergleuten mit und ohne Silikose im Vergleich zu nichtstaubexponierten Arbeitern. Int Arch Gewerbepath Gewerbehyg 1967; 23: $32-48$

${ }^{69}$ Ulmer WT, Reichel G, Werner U. Die chronisch obstruktive Bronchitis des Bergmannes. Untersuchungen zur Häufigkeit bei der Normalbevölkerung und bei Bergleuten. Die Bedeutung der Staubbelastung und der Einfluss des Rauchens. Int Arch Gewerbepath Gewerbehyg 1968; $25: 75-98$

70 Valentin H, Kann J. Zur Klinik und Begutachtung der Atmungsstörungen unter besonderer Berücksichtigung der chronischen Verschleißkrankheiten der Lungen und der Silikose. Berufskrankheiten in der keramischen und Glas-Industrie.; Heft 12 Würzburg, 1962

71 Vallyathan V, Brower PS, Green FHY et al. Radiographic and pathologic correlation of coal workers' pneumoconiosis. Am J Respir Crit Care Med 1996; 154: 741 - 748

72 Vallyathan V, Green FHY, Brower PS et al. The role of coal mine dust exposure in the development of pulmonary emphysema. Ann Occup Hyg 1997; 41: 352 - 357 (Inhaled Particles VIII)

73 Wang ML, Petsonk EL, Beckmann LA et al. Clinically important FEV declines among coal miners: an exploration of previously unrecognised determinants. Occup Environ Med 1999; 56: 837 - 844

74 Woitowitz HJ. Erkrankungen der Atemwege und der Lungen, des Rippenfells und des Bauchfells. In: Arbeitsmedizin. Kurzgefasstes Lehrbuch für Ärzte und Studenten. 2. Band. Stuttgart, New York: Thieme, 1985: $172-307$ 